\title{
Adaptive Algorithm for Chirp-Rate Estimation
}

\author{
Igor Djurović, ${ }^{1}$ Cornel Ioana (EURASIP Member), ${ }^{2}$ Ljubiša Stanković, ${ }^{1}$ and Pu Wang ${ }^{3}$ \\ ${ }^{1}$ Electrical Engineering Department, University of Montenegro, Cetinjski put bb, 81000 Podgorica, Montenegro \\ ${ }^{2}$ Gipsa Lab, INP Grenoble, 38402 Grenoble, France \\ ${ }^{3}$ Department of Electrical and Computer Engineering, Stevens Institute of Technology, Hoboken, NJ 07030, USA
}

Correspondence should be addressed to Igor Djurović, igordj@ac.me

Received 5 March 2009; Accepted 26 June 2009

Recommended by Vitor Nascimento

\begin{abstract}
Chirp-rate, as a second derivative of signal phase, is an important feature of nonstationary signals in numerous applications such as radar, sonar, and communications. In this paper, an adaptive algorithm for the chirp-rate estimation is proposed. It is based on the confidence intervals rule and the cubic-phase function. The window width is adaptively selected to achieve good tradeoff between bias and variance of the chirp-rate estimate. The proposed algorithm is verified by simulations and the results show that it outperforms the standard algorithm with fixed window width.
\end{abstract}

Copyright () 2009 Igor Djurović et al. This is an open access article distributed under the Creative Commons Attribution License, which permits unrestricted use, distribution, and reproduction in any medium, provided the original work is properly cited.

\section{Introduction}

Instantaneous frequency (IF) estimation is a challenging topic in the signal processing [1]. The IF is defined as the first derivative of the signal's instantaneous phase. Time-frequency (TF) representations are main tools for nonparametric IF estimation. The positions of peaks in the TF representation can be used as an IF estimator. There are several sources of errors in this estimator: higher-order derivatives of the signal phase and the noise. For relatively high signal-to-noise ratio (SNR), Stanković and Katkovnik have proposed an IF estimator based on intersection of confidence intervals rule (ICI) that produces results close to the optimal mean squared error (MSE) of the IF estimate, by achieving tradeoff between bias and variance [2-7].

Sometimes in practice there is a need for an estimation of the second-order derivative of signal phase. Estimation of this parameter, referred to as the chirp-rate, is important in radar systems, for example, focusing of the SAR images $[8,9]$.

Recently, O'Shea et al. have proposed a chirp-rate estimator based on the cubic phase function $(\mathrm{CPF})[10$ 14]. It gives accurate results for a third-order polynomial phase signal. In this paper, we consider nonparametric chirprate estimation without the assumption on the polynomial phase structure. To this end, an adaptive algorithm for the chirp-rate estimation is proposed based on the ICI algorithm
[15-18]. The proposed estimator performs well for moderate noise environments.

The paper is organized as follows. The CPF-based nonparametric chirp-rate estimator is presented in Section 2. In Section 3 asymptotic expressions for the bias and the variance of the nonparametric chirp-rate estimate are provided as a prerequisite for the proposed adaptive algorithm. Full details of the adaptive algorithm based the ICI principle are presented in Section 4. Numerical examples are given in Section 5. Conclusions are given in Section 6.

\section{CPF-based Nonparametric Chirp-Rate Estimator}

Consider a signal $f(t)=A \exp (j \phi(t))$. The first derivative of the signal phase, $\omega(t)=\phi^{\prime}(t)$, is the IF. An important group of the IF estimators is based on TF representations $[1,19,20]$. Consider, for example, the Wigner distribution (WD) in a windowed (pseudo) discrete-time form:

$$
\begin{aligned}
\mathrm{WD}_{h}(t, \omega)= & \sum_{n=-\infty}^{\infty} w_{h}(n T) \\
& \times f(t+n T) f^{*}(t-n T) \exp (-j 2 \omega n T),
\end{aligned}
$$


where $T$ is the sampling interval and $w_{h}(n T)$ is the window function of the width $h, w_{h}(t) \neq 0$ for $|t| \leq h / 2$. The IF can be estimated from locations of peaks in the WD as

$$
\widehat{\omega}_{h}(t)=\arg \max _{\omega} \mathrm{WD}_{h}(t, \omega) .
$$

A close look at the phase of the local autocorrelation $f(t+$ $n T) f^{*}(t-n T)$ by means of Taylor expansions is

$$
\begin{aligned}
& \Phi(t, n T) \\
& \quad=\phi(t+n T)-\phi(t-n T) \\
& \quad \approx 2 \phi^{\prime}(t)(n T)+\phi^{(3)}(t) \frac{(n T)^{3}}{3}+\phi^{(5)}(t) \frac{2(n T)^{5}}{5 !}+\cdots,
\end{aligned}
$$

where $\phi^{(k)}(t)$ is defined as the $k$ th derivative of the phase. When higher-order phase derivatives are equal to 0 , the WD is ideally concentrated along the IF, that is, it achieves maximum along the IF line $\omega(t)=\phi^{\prime}(t)$. Therefore, the IF can be calculated as

$$
\phi^{\prime}(t) \approx \frac{\phi(t+n T)-\phi(t-n T)}{2(n T)}
$$

by ignoring higher-order derivatives.

Estimation of the higher-order phase terms is also very important, for example, in radar signal processing (proper estimation of higher-order phase terms can be helpful in focusing of radar images [21-29]). Commonly, higher-order nonlinearity exists in the estimate. The nonlinearity causes performance degradation of the IF estimate. For example, it reduces the SNR threshold of the method applicability [23].

Analogy to the above observations on the IF estimation, the chirp-rate parameter (i.e., the second-derivative of the phase) can be obtained by

$$
\phi^{(2)}(t) \approx \frac{\phi(t+n T)-2 \phi(t)+\phi(t-n T)}{2(n T)^{2}} .
$$

This approximate formula corresponds to the local autocorrelation function $f(t+n T) f^{* 2}(t) f(t-n T)$. Since $f^{* 2}(t)$ does not depend on $n T$, the CPF was proposed for the chirp-rate estimation:

$$
\begin{aligned}
C_{h}(t, \Omega)= & \sum_{n=-\infty}^{\infty} w_{h}(n T) \\
& \times f(t+n T) f(t-n T) \exp \left(-j \Omega(n T)^{2}\right)
\end{aligned}
$$

where $\Omega$ denotes chirp-rate index. The rectangular window function (finite number of samples) is inherently assumed in the original O'Shea estimator. Here, in our derivations of the adaptive chirp-rate estimator, we will assume that a general window function is used. The CPF-based nonparametric chirp-rate estimation can be performed as

$$
\widehat{\Omega}_{h}(t)=\arg \max _{\Omega}\left|C_{h}(t, \Omega)\right|^{2} .
$$

In this manner, the nonlinearity of the chirp-rate estimation is kept to the same order as in the WD case, that is, the second, order nonlinearity. It results in high accuracy approaching the Cramer-Rao lower bound (CRLB) for a wide range of the SNR for Gaussian noise environment $[10,11,13]$.

However, nonpolynomial phase signal or high-order polynomial phase signal this estimator is biased, and the performance degrades. To relax the application range of the CPF-based chirp-rate estimator, in this following, an CPFbased algorithm with adaptive window width is proposed. Specifically, the window width is adaptively determined by using the ICI algorithm.

\section{Asymptotic Bias and Variance}

The chirp-rate is estimated by using the position of the peaks in the magnitude-squared CPF. The CPF is ideally concentrated on the chirp-rate for signals, when the fourthand other higher-order phase derivatives are equal to zero. However, for signals with these derivatives being different from zero, this is not the case. Higher-order derivatives produce bias in the chirp-rate estimation. The asymptotic expression for the bias, derived in the appendix, is

$$
\operatorname{bias}\left\{\hat{\Omega}_{h}(t)\right\}=E\left\{\Delta \Omega_{h}(t)\right\} \simeq \phi^{(4)}(t) w_{b} h^{2},
$$

where $w_{b}$ is a constant dependent on the selected window type only, while $\phi^{(4)}(t)$ is the fourth derivative of the signal phase. Assume that the signal corrupted by the additive white Gaussian noise $\nu(t)$ with

(i) mutually independent real and imaginary parts,

(ii) zero-mean $E\{\nu(t)\}=0$,

(iii) covariance $E\left\{\nu\left(t^{\prime}\right) \nu^{*}\left(t^{\prime \prime}\right)\right\}=\sigma^{2} \delta\left(t^{\prime}-t^{\prime \prime}\right)$, where $\sigma^{2}$ is variance while $\delta(t)$ is the Dirac delta function defined $\delta(t)=1$ for $t=0$ and $\delta(t)=0$ elsewhere.

Then, the asymptotic expression for variance of the chirp-rate estimator (7), for relatively high SNR, exhibits

$$
\operatorname{var}\left\{\hat{\Omega}_{h}(t)\right\} \simeq \frac{\sigma^{2}}{A^{2}} h^{-5} w_{v}
$$

where $w_{v}$ depends on the selected window type only (see appendix). Obviously, the bias increases with the increase of the window width, while the variance decreases at the same time. The MSE of the estimator is

$$
\begin{aligned}
\operatorname{MSE}\left\{\hat{\Omega}_{h}(t)\right\} & =\operatorname{bias}^{2}\left\{\hat{\Omega}_{h}(t)\right\}+\operatorname{var}\left\{\hat{\Omega}_{h}(t)\right\} \\
& =\left[\phi^{(4)}(t)\right]^{2} w_{b}^{2} h^{4}+\frac{\sigma^{2}}{A^{2}} h^{-5} w_{v} .
\end{aligned}
$$

From (10), by minimizing the MSE with respect to $h$, we get

$$
h_{\mathrm{opt}}(t)=\sqrt[9]{\frac{5\left(\sigma^{2} / A^{2}\right) w_{h}}{4\left[\phi^{(4)}(t)\right]^{2} w_{b}^{2}}} .
$$

Since the fourth-order derivative of the signal phase is not known in advance, we cannot determine the optimal 


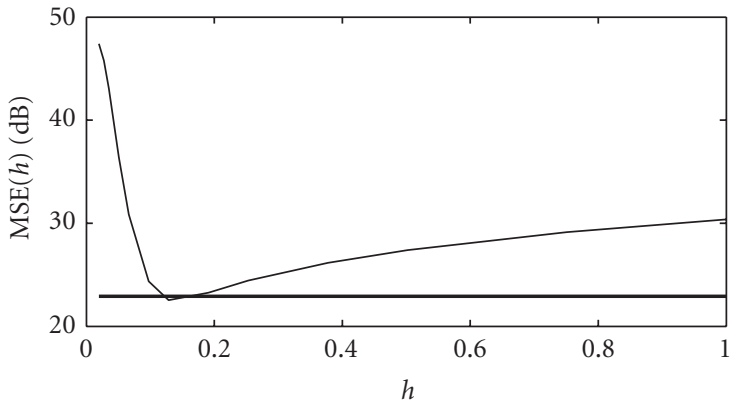

(a)

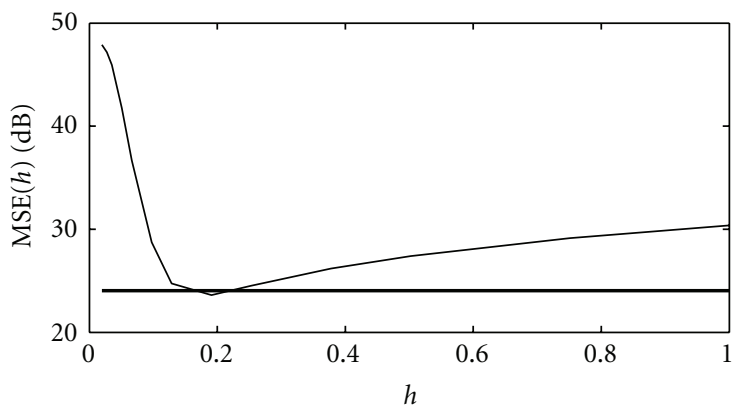

(c)

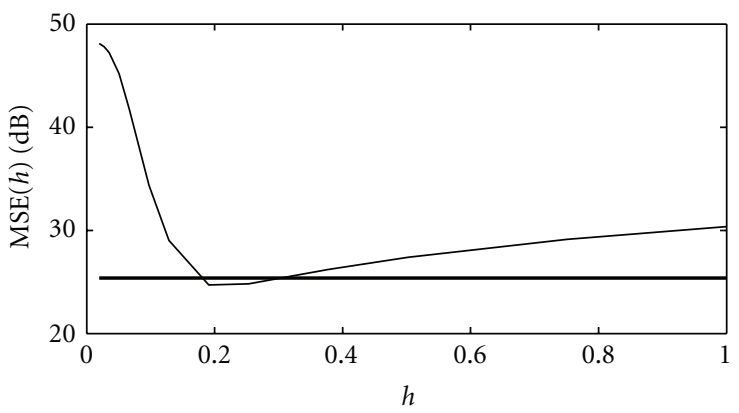

(e)

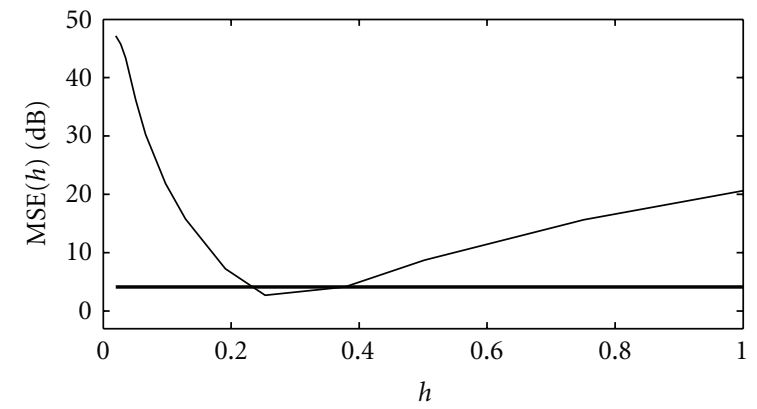

(b)

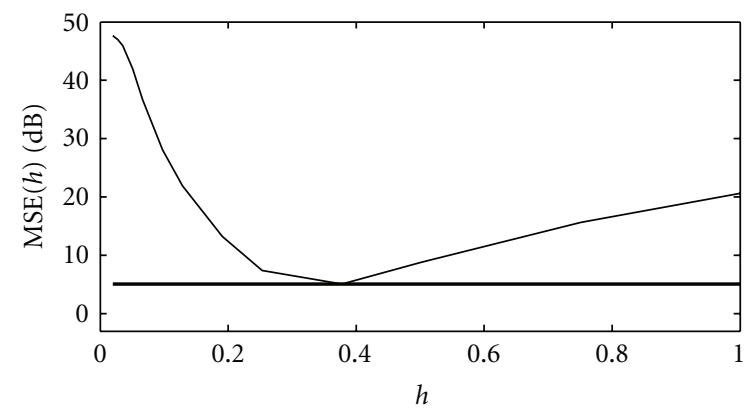

(d)

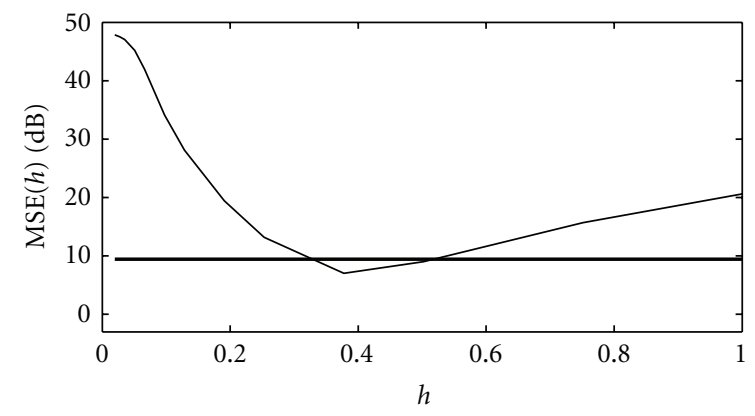

(f)

FIGURE 1: MSE for the chirp-rate estimation: (a) signal 1, $\sigma=0.06$; (b) signal 2, $\sigma=0.06$; (c) signal 1, $\sigma=0.09$; (d) signal 2, $\sigma=0.09$; (e) signal $1, \sigma=0.12$; (f) signal 2, $\sigma=0.12$, Thin line - fixed window estimator; thick line adaptive window width.

window length $h_{\mathrm{opt}}(t)$ in practice. In this paper, an algorithm that can produce adaptive window width, close to the optimal one, is proposed without knowing phase derivatives in advance. The ICI algorithm [2-7] is developed for similar problems with a tradeoff in parameter selection between the bias and variance. The ICI-based algorithm for the second-order derivative estimation is given in the following section.

\section{Intersection Confidence Interval Algorithm}

Here, we will briefly describe the ICI algorithm for achieving the tradeoff between influence of the higher-order derivatives (bias) and noise (variance). Consider the set of increasing window widths $H=\left\{h_{1}, h_{2}, \ldots, h_{Q}\right\}, h_{i}<h_{i+1}$. These windows are selected in such a manner that $h_{i} \approx a^{i-1} h_{1}$, $a>1$. It is assumed that the optimal window $h_{\mathrm{opt}}(t)$, for a given instant, is close to a value from the considered set.
Chirp-rate estimates corresponding to all windows from $H$ are $\widehat{\Omega}_{h_{i}}(t), i=1,2, \ldots, Q$. They are obtained as

$$
\widehat{\Omega}_{h_{i}}(t)=\arg \max _{\Omega}\left|C_{h_{i}}(t, \Omega)\right|^{2},
$$

where $C_{h_{i}}(t, \Omega)$ is the CPF calculated with window $w_{h_{i}}(t)$ of the width $h_{i}, w_{h_{i}}(t) \neq 0$ for $|t| \leq h_{i} / 2$. Around any estimate, we can create a confidence interval $\left[\widehat{\Omega}_{h_{i}}(t)-\kappa \sigma\left(h_{i}\right), \widehat{\Omega}_{h_{i}}(t)+\right.$ $\left.\kappa \sigma\left(h_{i}\right)\right]$, where $\kappa$ is the parameter that controls probability that exact chirp-rate parameter belongs to the interval, while $\sigma\left(h_{i}\right)=(\sigma / A) h_{i}^{-5 / 2} \sqrt{w_{v}}$ (A.2). For Gaussian variable we know that exact value of the parameter belongs to the interval with probability $P(\kappa)$ (e.g., $P(2)=0.95$ and $P(3)=0.997)$.

According to [7], the optimal window is close to the widest one where the confidence intervals, created with two neighboring windows from set $H$, still intersect. This can be written as

$$
\left|\widehat{\Omega}_{h_{i}}(t)-\widehat{\Omega}_{h_{i-1}}(t)\right| \leq \kappa\left(\sigma\left(h_{i}\right)+\sigma\left(h_{i-1}\right)\right) .
$$




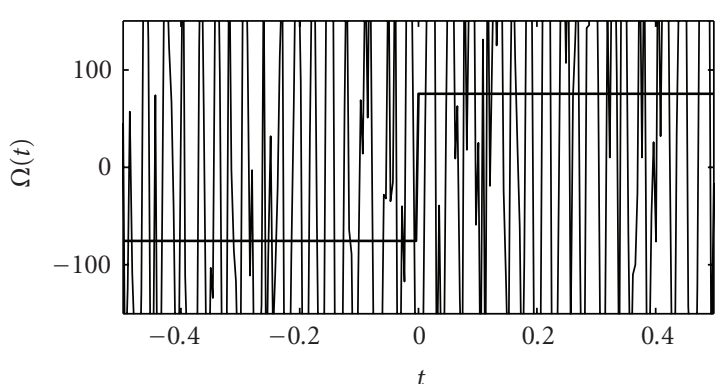

(a)

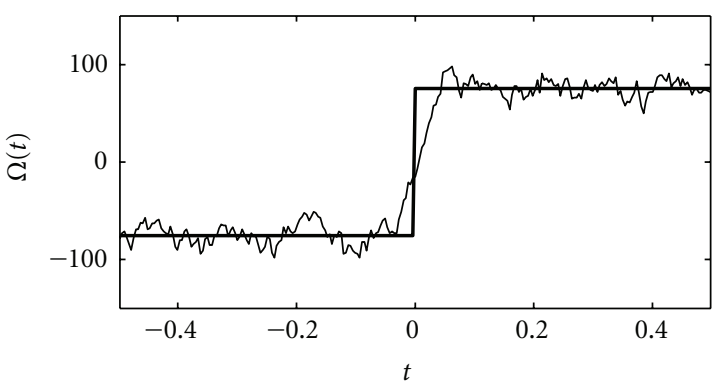

(c)

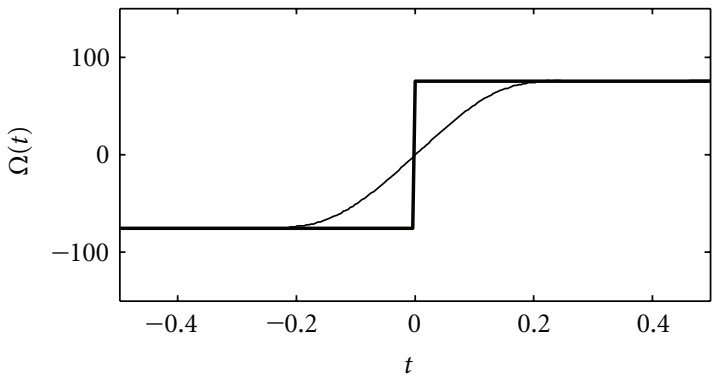

(e)

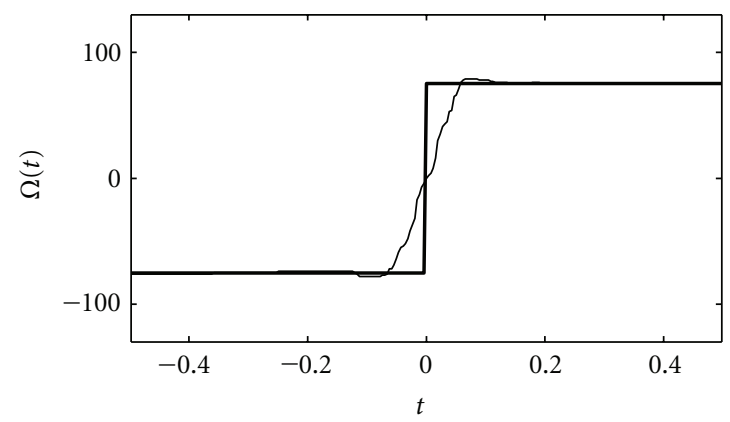

(g)

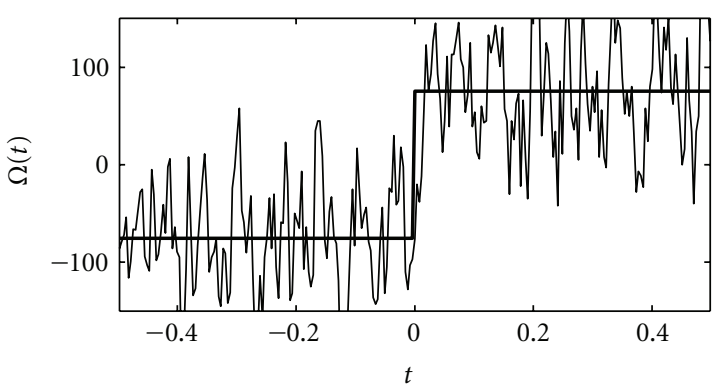

(b)

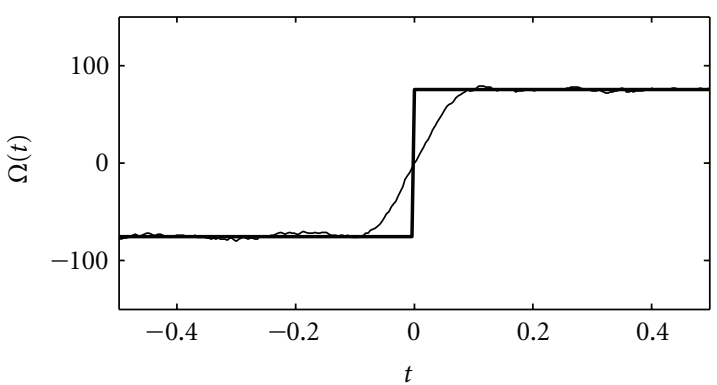

(d)

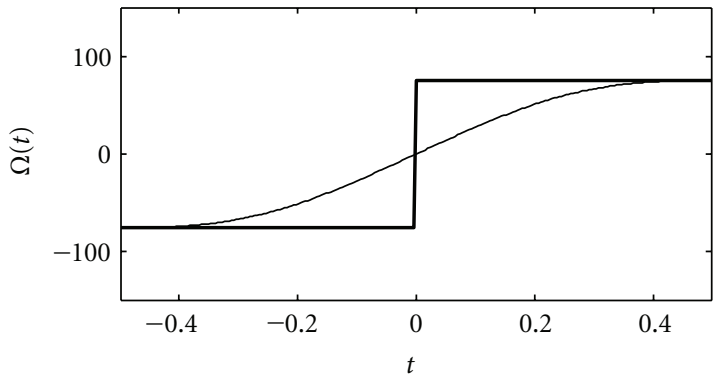

(f)

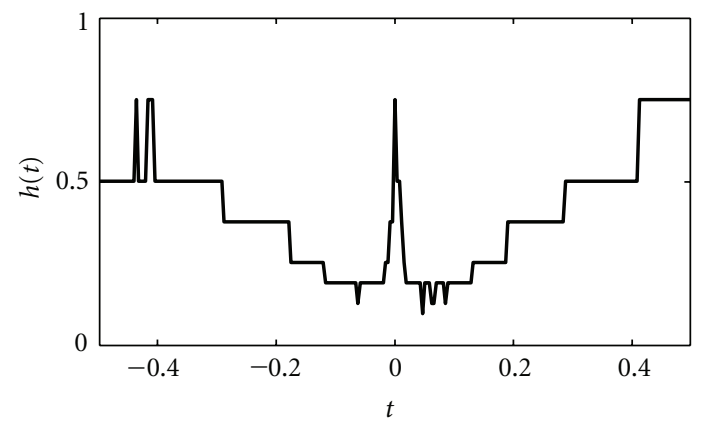

(h)

Figure 2: Chirp-rate estimation for test signal 1: (a) Fixed window $N=9$ samples $(h=9 / 257)$; (b) Fixed window $N=17$ samples ( $h=17 / 257)$; (c) Fixed window $N=33$ samples $(h=33 / 257)$; (d) Fixed window $N=65$ samples $(h=65 / 257)$; (e) Fixed window $N=129$ samples $(h=129 / 257)$; (f) Fixed window $N=257$ samples $(h=1)$; (g) Estimator with adaptive window width; (h) Adaptive window width.

It is required that this relationship holds also for all narrower windows:

$$
\left|\hat{\Omega}_{h_{j}}(t)-\hat{\Omega}_{h_{j-1}}(t)\right| \leq \kappa\left(\sigma\left(h_{j}\right)+\sigma\left(h_{j-1}\right)\right) \quad j \leq i .
$$

Then we can adopt that the optimal window estimate for the considered instant is $\hat{h}_{\text {opt }}(t)=h_{i}$ or $\hat{h}_{\text {opt }}(t)=h_{i-1}$.
As it is shown in [2], selection of particular window depends on bias and variance (in fact on powers of parameter of interest $h^{n}$ and $\left.h^{-m}\right)$ in considered application. Namely, in our application $\operatorname{bias}^{2}\left\{\hat{\Omega}_{h}(t)\right\} \propto h^{4}$ while $\operatorname{var}\left\{\hat{\Omega}_{h}(t)\right\} \propto$ $h^{-5}$. Then, according to [2], it is better to take previous window $\hat{h}_{\mathrm{opt}}(t)=h_{i-1}$ as the optimal estimate since the next window can already have large bias. The algorithm 


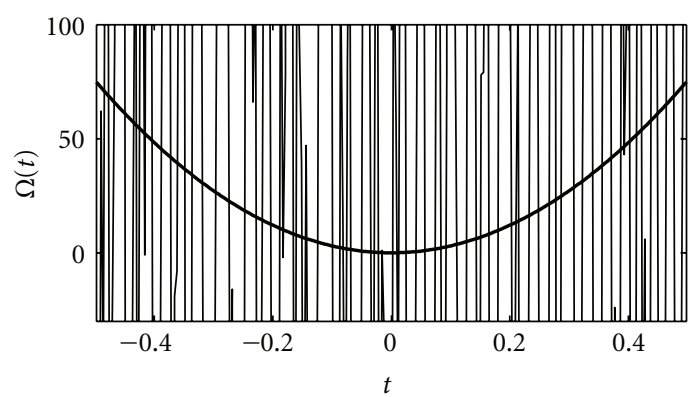

(a)

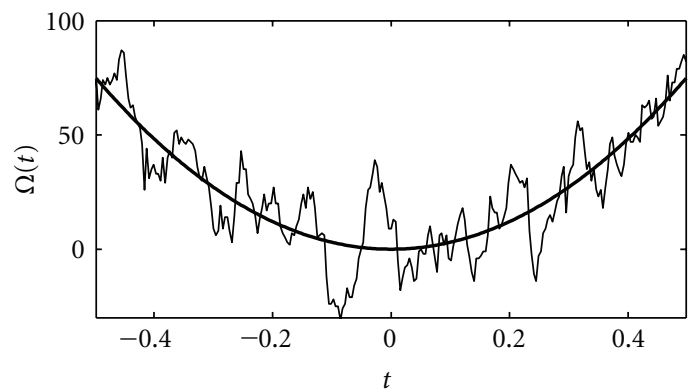

(c)

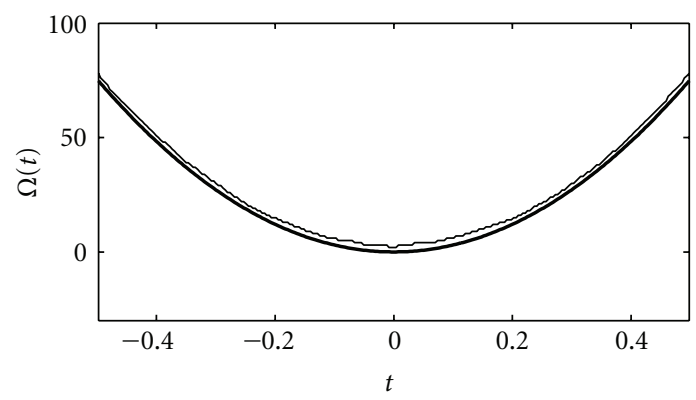

(e)

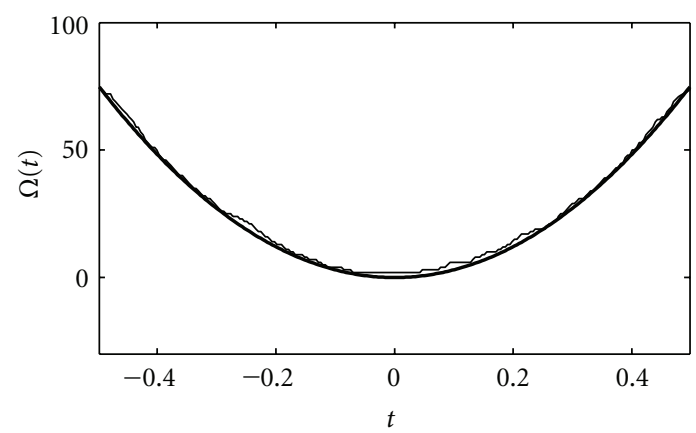

(g)

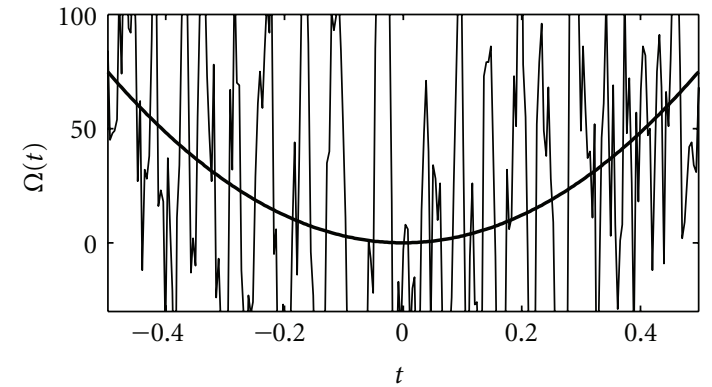

(b)

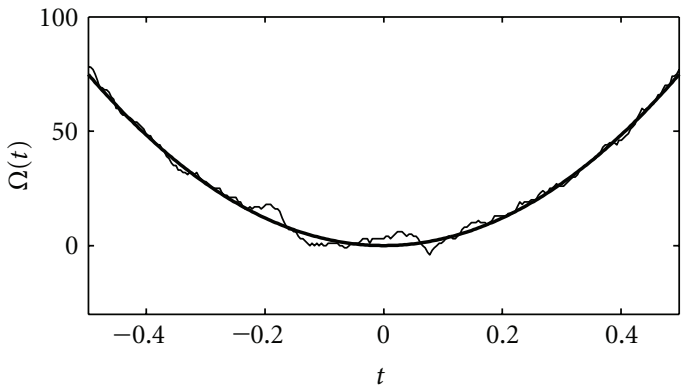

(d)

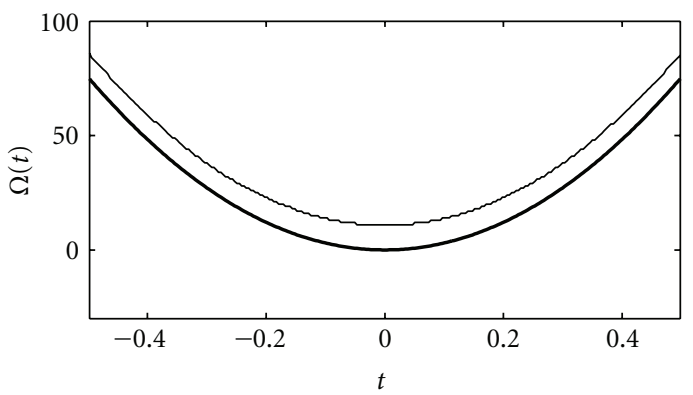

(f)

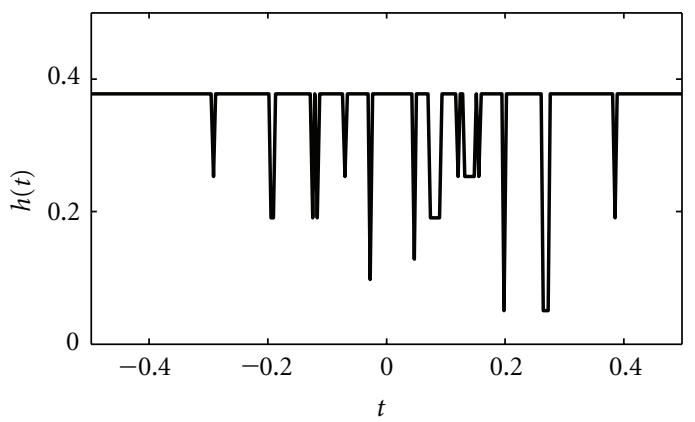

(h)

Figure 3: Chirp-rate estimation for test signal 2: (a) Fixed window $N=9$ samples $(h=9 / 257)$; (b) Fixed window $N=17$ samples ( $h=17 / 257)$; (c) Fixed window $N=33$ samples $(h=33 / 257)$; (d) Fixed window $N=65$ samples $(h=65 / 257)$; (e) Fixed window $N=129$ samples $(h=129 / 257)$; (f) Fixed window $N=257$ samples $(h=1)$; (g) Estimator with adaptive window width; (h) Adaptive window width.

accuracy depends on the proper selection of parameter $\kappa$. This selection is discussed in details in [2]. It can be assumed that the algorithm for relatively wide region of $\kappa \in[2,5]$ produces results of the same order of accuracy. The crossvalidation algorithm [4] or results from analysis given in [2] can be employed in the case where precise selection of this parameter is required. In our simulations, $\kappa=3$ is used.

The remaining question in the algorithm is how to estimate $\sigma\left(h_{i}\right)$ since the signal amplitude and noise variance $(A$ and $\sigma)$ are not known in advance. There are several 
approaches in literature, but here we will use a simple and very accurate technique from [30]. Namely, amplitude can be estimated as

$$
\widehat{A}^{2}=\sqrt{\left|2 M_{2}^{2}-M_{4}\right|}
$$

where

$$
M_{i}=\frac{1}{N} \sum x^{i}(n)
$$

where $N$ is number of signal samples, while the variance can be estimated as

$$
\widehat{\sigma}^{2}=\left|M_{2}-\widehat{A}^{2}\right|
$$

\section{Numerical Examples}

We considered two test signals:

$$
\begin{aligned}
& f_{1}(t)= \begin{cases}\exp \left(j 12 \pi t^{2}\right) & t \geq 0 \\
\exp \left(-j 12 \pi t^{2}\right) & t<0\end{cases} \\
& f_{2}(t)=\exp \left(j 8 \pi t^{4}\right) .
\end{aligned}
$$

The exact chirp-rates for these two signals are $\Omega_{1}(t)=24 \pi$ $\operatorname{sign}(t)$ and $\Omega_{2}(t)=96 \pi t^{2}$. Signal is considered within interval $t \in[-1 / 2,1 / 2]$ with sampling rate $T=1 / 257$. A set of used window widths is $h_{i}=N_{i} T$, where $N_{i}=a^{i-1} N_{1}$ and $a=\sqrt{2}$ and $N_{1}=5$. We always set the closest possible window from the set with odd number of samples in the interval. Total number of windows in the set is 13 . Figure 1 depicts the MSE of the obtained chirp-rate estimators for $\sigma=0.06$ (first row, SNR $=24 \mathrm{~dB}$ ), $\sigma=0.09$ (second row, $\mathrm{SNR}=21 \mathrm{~dB}$ ) and $\sigma=0.12$ (third row, SNR $=18 \mathrm{~dB}$ ). The left column is given for the first test signal (18) while the right column represents results for the second test signal (19). Results are obtained with the Monte Carlo simulation with 100 trials. Thin line marks results obtained with the windows of the fixed width, while thick line represents results achieved with the proposed algorithm. It can be seen that the proposed algorithm gives more accurate results than almost all windows with fixed width. It may happen that some of windows with fixed width outperform our algorithm, but it should be kept in mind that the best window is not known in advance. For example, it can be seen that the best fixed window width for the first test signal and $\sigma=0.06$ (Figure $1(\mathrm{a})$ ) is about $N=20$ samples, for the second signal and the same noise, it is about $N=50$ samples (Figure 1(b)), while for the first signal and $\sigma=0.12$ (Figure 1(e)), it is about $N=70$ samples.

Illustration of the adaptive CPF for the chirp-rate estimation for the first test signal embedded in the noise with $\sigma=0.09$ is depicted in Figure 2. Figures 2(a)-2(f) represent the result obtained with fixed window widths $(N=9, N=17, N=33, N=65, N=129$, and $N=257)$. Results obtained with the proposed algorithm are presented in Figure 2(g). Bias in the region close to the abrupt change can be observed. It is caused by the fact that we need a narrow window in this region and that this window produces estimate highly corrupted by noise (see Figure 2(a)). Figure 2(h) depicts the adaptive window width.

Results achieved with the second test signal for $\sigma=0.09$ are depicted in Figure 3. Here, the fourth order derivative of the signal phase is constant and we can expect that the optimal window width is constant. High noise influence can be observed for small window widths (Figures 3(b) and 3(c), $N=9$ and $N=17$ ) while, at the same time, the bias can be seen for wide window (Figure $3(\mathrm{f}), N=257$ ). The chirprate estimate and corresponding adaptive window width are depicted in Figures 3(g) and 3(h). It can be seen that the proposed algorithm gives adaptive window width close to constant as it was expected.

\section{Conclusion}

An adaptive chirp-rate estimator is introduced for a general signal model. It is based on the confidence intervals-rule. Selection of the algorithm parameters is discussed. The proposed algorithm is tested on two characteristic test signals. The obtained results are good, close to the optimal one that can be achieved with the CPF function.

\section{Appendices}

\section{A. Asymptotic Bias and Variance}

Our observation is modeled as $x(t)=f(t)+v(t)$ where $f(t)=A \exp (j \phi(t))$, while $v(t)$ is Gaussian noise with mutually independent real and imaginary parts, with zeromean $E\{\nu(t)\}=0$ and $E\left\{\nu\left(t^{\prime}\right) \nu^{*}\left(t^{\prime \prime}\right)\right\}=\sigma^{2} \delta\left(t^{\prime}-t^{\prime \prime}\right)$. Chirprate is estimated by using position of the CPF maximum. The $\mathrm{CPF}$ is ideally concentrated on the chirp-rate for noiseless signals when $\phi^{(k)}(t)=0$ for $k>3$. Introduce the following notation $F_{h}(t, \Omega)=\left|C_{h}(t, \Omega)\right|^{2}$ for squared-magnitude of the CPF. Here, index $h$ denotes width of the used even window function, $w_{h}(t) \neq 0$ for $|t| \leq h / 2, w_{h}(t)=w_{h}(-t)$. Two main sources of errors in the CPF are (1) errors caused by nonzero higher-order derivatives of the signal phase (contributing to the bias); (2) errors caused by the noise (contributing to the variance). For the sake of brevity, here we will give the main steps of the derivations. According to [3], the bias of the chirp-rate estimator can be expressed as

$$
E\left\{\Delta \Omega_{h}(t)\right\}=\operatorname{bias}\left\{\hat{\Omega}_{h}(t)\right\}=-\frac{\left.\left(\partial F_{h}(t, \Omega) / \partial \Omega\right)\right|_{0 \delta_{\Omega}}}{\left.\left(\partial^{2} F_{h}(t, \Omega) / \partial \Omega^{2}\right)\right|_{0}},
$$

while the variance is

$$
\operatorname{var}\left\{\hat{\Omega}_{h}(t)\right\}=\frac{E\left\{\left[\left.\left(\partial F_{h}(t, \Omega) / \partial \Omega\right)\right|_{0 \delta_{\nu}}\right]^{2}\right\}}{\left[\left.\left(\partial^{2} F_{h}(t, \Omega) / \partial \Omega^{2}\right)\right|_{0}\right]^{2}}
$$

where the following hold:

(i) $\partial^{2} F_{h}(t, \Omega) /\left.\partial \Omega^{2}\right|_{0}$ is evaluated at the position of the true chirp-rate, with the assumption that the signal has all phase derivatives higher than 2 equal to zero and that there is no noise; 
(ii) $\partial F_{h}(t, \Omega) /\left.\partial \Omega\right|_{0 \delta_{\Delta \Omega}}$ is evaluated at the position of true chirp-rate with assumption that estimation error is caused only by higher-order derivatives of the signal phase (noise-free assumption);

(iii) $\partial F_{h}(t, \Omega) /\left.\partial \Omega\right|_{0 \delta_{v}}$ is evaluated at the position of the true chirp-rate with the assumption that there is no higher order phase derivatives, that is, noise only influenced error.

Then three intermediate quantities $\left.\left(\partial^{2} F_{h}(t, \Omega) / \partial \Omega^{2}\right)\right|_{0}$, $\left.\left(\partial F_{h}(t, \Omega) / \partial \Omega\right)\right|_{0 \delta_{\Delta \Omega}}$, and $E\left\{\left[\left.\left(\partial F_{h}(t, \Omega) / \partial \Omega\right)\right|_{0 \delta_{\nu}}\right]^{2}\right\}$ are needed to determine asymptotic bias and variance. Calculations of these quantities are shown below.

A.1. Determination of $\partial^{2} F_{h}(t, \Omega) /\left.\partial \Omega^{2}\right|_{0}$. Determination of $\partial^{2} F_{h}(t, \Omega) /\left.\partial \Omega^{2}\right|_{0}$ is performed on true chirp-rate, that is, $\Omega=\phi^{(2)}(t)$ under assumption that there is noise and higherorder terms in the signal phase. Then the CPF exhibits

$$
\begin{aligned}
C_{h}(t, \Omega)= & \exp (j 2 \phi(t)) \sum_{n=-\infty}^{\infty} w_{h}(n T) A^{2} \\
& \times \exp \left(j \phi^{(2)}(t)(n T)^{2}\right) \\
& \times \exp \left(-j \Omega(n T)^{2}\right) .
\end{aligned}
$$

Value of $F_{h}(t, \Omega)=\left|C_{h}(t, \Omega)\right|^{2}$ is

$$
\begin{aligned}
F_{h}(t, \Omega)= & A^{4} \sum_{n_{1}=-\infty}^{\infty} \sum_{n_{2}=-\infty}^{\infty} w_{h}\left(n_{1} T\right) w_{h}^{*}\left(n_{2} T\right) \\
& \times \exp \left(j \phi^{(2)}(t)\left(n_{1} T\right)^{2}-j \phi^{(2)}(t)\left(n_{2} T\right)^{2}\right) \\
& \times \exp \left(-j \Omega\left(n_{1} T\right)^{2}+j \Omega\left(n_{2} T\right)^{2}\right) .
\end{aligned}
$$

The second partial derivative $\partial^{2} F_{h}(t, \Omega) /\left.\partial \Omega^{2}\right|_{0}$, evaluated for $\Omega=\phi^{(2)}(t)$, is

$$
\begin{aligned}
& \left.\frac{\partial^{2} F_{h}(t, \Omega)}{\partial \Omega^{2}}\right|_{0} \\
& =-\sum_{n_{1}} \sum_{n_{2}} A^{4} w_{h}\left(n_{1} T\right) w_{h}^{*}\left(n_{2} T\right) \\
& \quad \times\left(\left(n_{1} T\right)^{2}-\left(n_{2} T\right)^{2}\right)^{2} \\
& =-2 A^{4} \sum \sum_{n_{1}} n_{2} w_{h}\left(n_{1} T\right) w_{h}\left(n_{2} T\right) \\
& \quad \times\left[\left(n_{1} T\right)^{4}-\left(n_{1} T\right)^{2}\left(n_{2} T\right)^{2}\right] \\
& =2 A^{4} h^{4}\left[F_{2}^{2}-F_{4} F_{0}\right],
\end{aligned}
$$

where (see [3, appendix])

$$
F_{k}=\int_{-1 / 2}^{1 / 2} w(t) t^{k} d t
$$

A.2. Determination of $\partial F_{h}(t, \Omega) /\left.\partial \Omega\right|_{0 \delta_{\Delta \Omega}}$. Assumptions in the evaluation of the second term $\partial F_{h}(t, \Omega) /\left.\partial \Omega\right|_{0 \delta_{\Delta \Omega}}$ are similar like for the first terms, except the influence of the higherorder phase terms that now is not neglected:

$$
\begin{aligned}
& \left.\frac{\partial F_{h}(t, \Omega)}{\partial \Omega}\right|_{0 \delta_{\Delta \Omega}} \\
& \quad=A^{4} \sum_{n_{1}} \sum_{n_{2}} w_{h}\left(n_{1} T\right) w_{h}^{*}\left(n_{2} T\right)\left(-j\left(\left(n_{1} T\right)^{2}-\left(n_{2} T\right)^{2}\right)\right) \\
& \quad \times \exp \left(2 j \sum_{k=2}^{\infty} \phi^{(2 k)}(t) \frac{\left(n_{1} T\right)^{2 k}-\left(n_{2} T\right)^{2 k}}{(2 k) !}\right) .
\end{aligned}
$$

For simplicity, all higher-order derivatives, except the fourth order are removed, that is, $\left|\phi^{(4)}(t)\right| \gg\left|\phi^{(2 k)}(t)\right|$ for $k>2$ :

$$
\begin{aligned}
& \left.\frac{\partial F_{h}(t, \Omega)}{\partial \Omega}\right|_{0 \delta_{\Delta \Omega}} \\
& \quad=A^{4} \sum_{n_{1}} \sum_{n_{2}} w_{h}\left(n_{1} T\right) w_{h}^{*}\left(n_{2} T\right)\left(-j\left(\left(n_{1} T\right)^{2}-\left(n_{2} T\right)^{2}\right)\right) \\
& \quad \times \exp \left(j \phi^{(4)}(t) \frac{\left(n_{1} T\right)^{4}-\left(n_{2} T\right)^{4}}{12}\right) .
\end{aligned}
$$

Under the assumption that argument of exponential function $\phi^{(4)}(t)\left(\left(\left(n_{1} T\right)^{4}-\left(n_{2} T\right)^{4}\right) / 12\right)$ is relatively small, we can write

$$
\begin{gathered}
\exp \left(j \phi^{(4)}(t) \frac{\left(n_{1} T\right)^{4}-\left(n_{2} T\right)^{4}}{12}\right) \\
\approx 1+j \phi^{(4)}(t) \frac{\left(n_{1} T\right)^{4}-\left(n_{2} T\right)^{4}}{12} .
\end{gathered}
$$

Finally, we get

$$
\begin{aligned}
& \left.\frac{\partial F_{h}(t, \Omega)}{\partial \Omega}\right|_{0 \delta_{\Delta \Omega}} \\
& =\phi^{(4)}(t) \sum_{n_{1}} \sum_{n_{2}} A^{4} w_{h}\left(n_{1} T\right) w_{h}^{*}\left(n_{2} T\right) \\
& \quad \times\left(\left(n_{1} T\right)^{2}-\left(n_{2} T\right)^{2}\right)\left(\left(n_{1} T\right)^{4}-\left(n_{2} T\right)^{4}\right) \\
& =2 A^{4} \phi^{(4)}(t) h^{6}\left[F_{6} F_{0}-F_{2} F_{4}\right] .
\end{aligned}
$$


A.3. Determination of $E\left\{\left[\partial F_{h}(t, \Omega) /\left.\partial \Omega\right|_{0 \delta_{v}}\right]^{2}\right\}$. In the evaluation of $E\left\{\left[\partial F_{h}(t, \Omega) /\left.\partial \Omega\right|_{0 \delta_{\nu}}\right]^{2}\right\}$ higher-order phase terms are removed while now we consider the influence of the additive Gaussiannoise. Then, the term required for determination of the variance is given as

$$
\begin{aligned}
& E\left\{\left[\left.\frac{\partial F_{h}(t, \Omega)}{\partial \Omega}\right|_{0 \delta_{v}}\right]^{2}\right\} \\
& =\sum_{n_{1}} \sum_{n_{2}} \sum_{n_{3}} \sum_{n_{4}} w_{h}\left(n_{1} T\right) w_{h}\left(n_{2} T\right) w_{h}\left(n_{3} T\right) w_{h}\left(n_{4} T\right) \\
& \quad \times E\left\{x\left(t+n_{1} T\right) x\left(t-n_{1} T\right) x^{*}\left(t+n_{2} T\right) x^{*}\left(t-n_{2} T\right)\right. \\
& \left.\quad \times x^{*}\left(t+n_{3} T\right) x^{*}\left(t-n_{3} T\right) x\left(t+n_{4} T\right) x\left(t-n_{4} T\right)\right\} \\
& \quad \times\left(\left(n_{1} T\right)^{2}-\left(n_{2} T\right)^{2}\right)\left(\left(n_{3} T\right)^{2}-\left(n_{4} T\right)^{2}\right) \\
& \quad \times \exp \left(-j \Omega\left(n_{1} T\right)^{2}+j \Omega\left(n_{2} T\right)^{2}+j \Omega\left(n_{3} T\right)^{2}-\Omega\left(n_{4} T\right)^{2}\right) .
\end{aligned}
$$

Determination of

$$
\begin{aligned}
& E\left\{x\left(t+n_{1} T\right) x\left(t-n_{1} T\right) x^{*}\left(t+n_{2} T\right) x^{*}\left(t-n_{2} T\right)\right. \\
& \left.\quad \times x^{*}\left(t+n_{3} T\right) x^{*}\left(t-n_{3} T\right) x\left(t+n_{4} T\right) x\left(t-n_{4} T\right)\right\}
\end{aligned}
$$

is a rather tedious job. By assuming high SNR, that is, $A^{2} / \sigma^{2} \gg 1$, (A.12) can be approximated by using only terms with two noise factors. Then, from all possible 128 combinations of signal and noise we can select just those where we have 2 noise terms and 6 signal terms. Namely, combinations with 1 and 3 noise terms give expectation equal to zero, while we can assume that combinations with 4 and more noise terms due to introduced high SNR assumption are much smaller than the expectation of combinations with 2 noise terms. There are 28 combinations in total, with 2 noise terms. Fortunately, a high number of them have zero expectation. Namely, for the used noise model (complex Gaussian noise with independent real and imaginary parts) it holds that $E\left\{v\left(t_{1}\right) \nu\left(t_{2}\right)\right\}=E\left\{\nu^{*}\left(t_{1}\right) \nu^{*}\left(t_{2}\right)\right\}=0$. This eliminates 12 combinations from (A.12). Furthermore, combinations $E\left\{\nu\left(t \pm n_{1} T\right) \nu^{*}\left(t \pm n_{2} T\right)\right\}=\sigma^{2} \delta\left(n_{1} \pm n_{2}\right)$ and combinations $E\left\{v^{*}\left(t \pm n_{3} T\right) \nu\left(t \pm n_{4} T\right)\right\}=\sigma^{2} \delta\left(n_{3} \pm n_{4}\right)$ will also produce a zero-mean, since they cause $\left(n_{1} T\right)^{2}-\left(n_{2} T\right)^{2}=$ 0 or $\left(n_{3} T\right)^{2}-\left(n_{4} T\right)^{2}=0$ in (A.11). This eliminates next 8 combinations. Only 8 remaining combinations, $E\{\nu(t \pm$ $\left.\left.n_{1} T\right) \nu^{*}\left(t \pm n_{3} T\right)\right\}=\sigma^{2} \delta\left(n_{1} \pm n_{3}\right)$ and $E\left\{\nu^{*}\left(t \pm n_{2} T\right) \nu(t \pm\right.$ $\left.\left.n_{4} T\right)\right\}=\sigma^{2} \delta\left(n_{2} \pm n_{4}\right)$, give results of interest. We will consider just one of these 8 combinations, since all others produce the same result. Here, we will consider situation where the first term $x\left(t+n_{1} T\right)$ and the fifth $x^{*}\left(t+n_{3} T\right)$ are noisy terms while others are signal terms:

$$
\begin{aligned}
\sum_{n_{1}} \sum_{n_{2}} \sum_{n_{3}} \sum_{n_{4}} w_{h}\left(n_{1} T\right) w_{h}\left(n_{2} T\right) w_{h}\left(n_{3} T\right) w_{h}\left(n_{4} T\right) \\
\quad \times \sigma^{2} \delta\left(n_{1}-n_{3}\right) f\left(t-n_{1} T\right) f^{*}\left(t+n_{2} T\right) f^{*}\left(t-n_{2} T\right) \\
\quad \times f^{*}\left(t+n_{3} T\right) f^{*}\left(t-n_{3} T\right) f\left(t+n_{4} T\right) f\left(t-n_{4} T\right) \\
\quad \times\left(\left(n_{1} T\right)^{2}-\left(n_{2} T\right)^{2}\right)\left(\left(n_{3} T\right)^{2}-\left(n_{4} T\right)^{2}\right) \\
\quad \times \exp \left(-j \Omega\left(n_{1} T\right)^{2}+j \Omega\left(n_{2} T\right)^{2}+j \Omega\left(n_{3} T\right)^{2}-\Omega\left(n_{4} T\right)^{2}\right) \\
=\sum_{n_{1}} \sum_{n_{2}} \sum_{n_{4}} \sigma^{2}\left|f\left(t-n_{1} T\right)\right|^{2} w_{h}^{2}\left(n_{1} T\right) w_{h}\left(n_{2} T\right) w_{h}\left(n_{4} T\right) \\
\quad \times f^{*}\left(t+n_{2} T\right) f^{*}\left(t-n_{2} T\right) f\left(t+n_{4} T\right) f\left(t-n_{4} T\right) \\
\quad \times\left(\left(n_{1} T\right)^{2}-\left(n_{2} T\right)^{2}\right)\left(\left(n_{1} T\right)^{2}-\left(n_{4} T\right)^{2}\right) \\
\quad \times \exp \left(j \Omega\left(n_{2} T\right)^{2}-\Omega\left(n_{4} T\right)^{2}\right) \\
=\sigma^{2} A^{6} \sum_{n_{1}} \sum_{n_{2}} \sum_{n_{4}} w_{h}^{2}\left(n_{1} T\right) w_{h}\left(n_{2} T\right) w_{h}\left(n_{4} T\right) \\
\quad \times\left(\left(n_{1} T\right)^{2}-\left(n_{2} T\right)^{2}\right)\left(\left(n_{1} T\right)^{2}-\left(n_{4} T\right)^{2}\right) \\
=\sigma^{2} A^{6} h^{3}\left[E_{4} F_{0}^{2}-2 E_{2} F_{2} F_{0}+E_{0} F_{2}^{2}\right],
\end{aligned}
$$

where $E_{k}$ is calculated according to [3]

$$
E_{k}=\frac{1}{T} \int_{-1 / 2}^{1 / 2} w^{2}(t) t^{k} d t
$$

The same results as (A.13) can be obtained for the other seven terms, so we have

$$
E\left\{\left[\left.\frac{\partial F_{h}(t, \Omega)}{\partial \Omega}\right|_{0 \delta_{v}}\right]^{2}\right\}=8 \sigma^{2} A^{6} h^{3}\left[E_{4} F_{0}^{2}-2 E_{2} F_{2} F_{0}+E_{0} F_{2}^{2}\right] .
$$

Substituting (A.5), (A.10), and (A.15) in (A.1) and (A.2), we are getting expressions for the bias and variance (8) and (9).

\section{Acknowledgments}

The work of I. Djurovic is realized at the INP Grenoble, France, and supported by the CNRS, under contract no. 180 089013 00387. The work of P. Wang was supported in part by the National Natural Science Foundation of China under Grant 60802062.

\section{References}

[1] L. Boashash, "Estimating and interpreting the instantaneous frequency of a signal-part I," Proceedings of the IEEE, vol. 80, no. 4, pp. 521-538, 1992.

[2] L. Stanković, "Performance analysis of the adaptive algorithm for bias-to-variance tradeoff," IEEE Transactions on Signal Processing, vol. 52, no. 5, pp. 1228-1234, 2004. 
[3] V. Katkovnik and L. Stanković, "Instantaneous frequency estimation using the Wigner distribution with varying and data-driven window length," IEEE Transactions on Signal Processing, vol. 46, no. 9, pp. 2315-2325, 1998.

[4] V. Katkovnik and L. Stanković, "Periodogram with varying and data-driven window length," Signal Processing, vol. 67, no. 3, pp. 345-358, 1998.

[5] L. Stanković and V. Katkovnik, "Algorithm for the instantaneous frequency estimation using time-frequency distributions with variable window width," IEEE Signal Processing Letters, vol. 5, no. 9, pp. 224-227, 1998.

[6] L. Stanković and V. Katkovnik, "Instantaneous frequency estimation using higher order distributions with adaptive order and window length," IEEE Transactions on Information Theory, vol. 46, no. 1, pp. 302-311, 2000.

[7] L. Stanković, "Adaptive instantaneous frequency estimation using TFDs," in Time-Frequency Signal Analysis and Processing, B. Boashash, Ed., Elsevier, New York, NY, USA, 2003.

[8] I. Djurović, T. Thayaparan, and L. Stanković, "SAR imaging of moving targets using polynomial Fourier transform," IET Signal Processing, vol. 2, no. 3, pp. 237-246, 2008.

[9] I. Djurović, T. Thayaparan, and L. Stanković, "Adaptive local polynomial Fourier transform in ISAR," EURASIP Journal on Applied Signal Processing, vol. 2006, Article ID 36093, 15 pages, 2006.

[10] P. O'Shea, "A fast algorithm for estimating the parameters of a quadratic FM signal," IEEE Transactions on Signal Processing, vol. 52, no. 2, pp. 385-393, 2004.

[11] M. Farquharson and P. O'Shea, "Extending the performance of the cubic phase function algorithm," IEEE Transactions on Signal Processing, vol. 55, no. 10, pp. 4767-4774, 2007.

[12] M. Farquharson, P. O'Shea, and G. Ledwich, "A computationally efficient technique for estimating the parameters of polynomial-phase signals from noisy observations," IEEE Transactions on Signal Processing, vol. 53, no. 8, pp. 3337-3342, 2005.

[13] P. O'Shea, "A new technique for instantaneous frequency rate estimation," IEEE Signal Processing Letters, vol. 9, no. 8, pp. 251-252, 2002.

[14] P. Wang, I. Djurović, and J. Yang, "Instantaneous frequency rate estimation based on the robust cubic phase function," in Proceedings of IEEE International Conference on Acoustics, Speech and Signal Processing (ICASSP '06), vol. 3, pp. 89-92, Toulouse, France, May 2006.

[15] V. Katkovnik, K. Egiazarian, and J. Astola, "Application of the ICI principle to window size adaptive median filtering," Signal Processing, vol. 83, no. 2, pp. 251-257, 2003.

[16] B. Krstajić, L. Stanković, and Z. Uskoković, "An approach to variable step-size LMS algorithm," Electronics Letters, vol. 38, no. 16, pp. 927-928, 2002.

[17] J. Astola, K. Egiazarian, and V. Katkovnik, "Adaptive window size image denoising based on ICI rule," in Proceedings of IEEE International Conference on Acoustics, Speech and Signal Processing (ICASSP '01), vol. 3, pp. 1869-1872, Salt Lake City, Utah, USA, May 2001.

[18] I. Djurović and L. Stanković, "Modification of the ICI rule-based IF estimator for high noise environments," IEEE Transactions on Signal Processing, vol. 52, no. 9, pp. 2655-2661, 2004.

[19] B. Barkat, "Instantaneous frequency estimation of nonlinear frequency-modulated signals in the presence of multiplicative and additive noise," IEEE Transactions on Signal Processing, vol. 49, no. 10, pp. 2214-2222, 2001.
[20] Z. M. Hussain and B. Boashash, "Adaptive instantaneous frequency estimation of multicomponent FM signals using quadratic time-frequency distributions," IEEE Transactions on Signal Processing, vol. 50, no. 8, pp. 1866-1876, 2002.

[21] T. J. Abatzoglou, "Fast maximum likelihood joint estimation of frequency and frequency rate," IEEE Transactions on Aerospace and Electronic Systems, vol. 22, no. 6, pp. 708-715, 1986.

[22] P. M. Djurić and S. Kay, "Parameter estimation of chirp signals," IEEE Transactions on Signal Processing, vol. 38, no. 12, pp. 2118-2126, 1990.

[23] S. Peleg and B. Porat, "Linear FM signal parameter estimation from discrete-time observations," IEEE Transactions on Aerospace and Electronic Systems, vol. 27, no. 4, pp. 607-616, 1991.

[24] J. C. Wood and D. T. Barry, "Radon transformation of time-frequency distributions for analysis of multicomponent signals," IEEE Transactions on Signal Processing, vol. 42, no. 11, pp. 3166-3177, 1994.

[25] S. Barbarossa, "Analysis of multicomponent LFM signals by a combined Wigner-Hough transform," IEEE Transactions on Signal Processing, vol. 43, no. 6, pp. 1511-1515, 1995.

[26] B. Friedlander and J. M. Francos, "Estimation of amplitude and phase parameters of multicomponent signals," IEEE Transactions on Signal Processing, vol. 43, no. 4, pp. 917-926, 1995.

[27] S. Peleg and B. Friedlander, "Multicomponent signal analysis using the polynomial-phase transform," IEEE Transactions on Aerospace and Electronic Systems, vol. 32, no. 1, pp. 378-387, 1996.

[28] S. Barbarossa, A. Scaglione, and G. B. Giannakis, "Product high-order ambiguity function for multicomponent polynomial-phase signal modeling," IEEE Transactions on Signal Processing, vol. 46, no. 3, pp. 691-708, 1998.

[29] L. Cirillo, A. Zoubir, and M. Amin, "Parameter estimation for locally linear FM signals using a time-frequency Hough transform," IEEE Transactions on Signal Processing, vol. 56, no. 9, pp. 4162-4175, 2008.

[30] S.-C. Sekhar and T. V. Sreenivas, "Signal-to-noise ratio estimation using higher-order moments," Signal Processing, vol. 86, no. 4, pp. 716-732, 2006. 